Научная статья

УДК 338

DOI: $10.17213 / 2075-2067-2021-6-196-205$

\title{
ФОРМИРОВАНИЕ СИСТЕМЫ ВЗАИМОДЕЙСТВИЯ ПРИОРИТЕТОВ УПРАВЛЕНИЯ ПОТЕНЦИАЛОМ ИННОВАЦИОННОЙ И ЧЕЛОВЕЧЕСКОЙ ДЕЯТЕЛЬНОСТИ
}

\author{
Наталия Игоревна Лаврикова ${ }^{1 凶}$, Наталья Анатольевна Азарова ${ }^{2}$, \\ Мария Васильевна Опара ${ }^{3}$
}

\author{
'Академия Федеральной службы охраны Российской Федерации, Орел, Россия \\ ${ }^{2,3}$ Воронежский государственный лесотехнический университет \\ имени Г. Ф. Морозова, Воронеж, Россия \\ ${ }^{1}$ nalavrikova@yandex.ru ${ }^{\bowtie}$, ORCID: 0000-0002-9459-2793, AuthorID РИНЦ: 534247 \\ 2azarovarsd@rambler.ru, ORCID: 0000-0001-8244-8922, AuthorID РИНЦ: 583069 \\ 3filichkinam@yandex.ru, ОRСІD: 0000-0002-3048-5358, AuthorID РИНЦ: 587010
}

Аннотация. Целью исследования является изучение процесса формирования системь комплексного исследования приоритетов управления потенциалом инновационной деятельности, а также изучение сложности понятия потенциала инновационной экономики.

Методологическая база исследования представляет собой системный эмпирический и структурно-функциональный подход рассмотрения объекта исследования в виде целостного комплекса взаимосвязанных элементов. К используемым научным методам относится анализ причинно-следственных связей.

Результаты исследования. Авторами определено, что понятие ресурсов как экономической категории нельзя отождествлять с понятием потенциала. В статье показана структура понятия потенциала инноваџионной деятельности, его элементы и измерения, а также способы формирования человеческого капитала, рассматриваемые как важнейшая составляющая обсуждаемого потенциала. Отсутствие структурированного теоретического обсуждения сущуности, смысла и способов формирования потенциала инноваџионной деятельности - важная проблема всей теории инновачий.

Перспективы исследования заключаются в изучении вопросов измерения инновационного и человеческого потенциала с учетом информационного потенџиала в целях реализаичии потребностей инновационной деятельности на различных уровнях управления.

Ключевые слова: инновационный потенциал, человеческий капитал, организационные возможности, управление

Для цитирования: Лаврикова Н.И., Азарова Н.А., Опара М.В. Формирование системь взаимодействия приоритетов управления потенциалом инновационной и человеческой деятельности // Вестник Южно-Российского государственного технического университета. Серия: Соџиально-экономические науки. 2021. T. 14, №6. C. 196-205. http://dx.doi. org/10.17213/2075-2067-2021-6-196-205.

(С) Лаврикова Н.И., Азарова Н.А., Опара М.В., 2021 
Original article

\title{
FORMATION OF A SYSTEM OF INTERACTION OF PRIORITIES FOR MANAGING THE POTENTIAL OF INNOVATION AND HUMAN ACTIVITY
}

\author{
Natalia I. Lavrikova ${ }^{1 凶}$, Natalya A. Azarova ${ }^{2}$, Maria V. Opara ${ }^{3}$ \\ ${ }^{1}$ Academy of the Federal Guard Service of the Russian Federation, Orel, Russia \\ ${ }^{2,3}$ Voronezh State University of Forestry and Technologies \\ named after G. F. Morozov, Voronezh, Russia \\ ${ }^{1}$ nalavrikova@yandex.ru ${ }^{\bowtie}$, ORCID: 0000-0002-9459-2793, AuthorID RSCI: 534247 \\ 2azarovarsd@rambler.ru, ORCID: 0000-0001-8244-8922, AuthorID RSCI: 583069 \\ 3ilichkinam@yandex.ru, ORCID:0000-0002-3048-5358, AuthorID RSCI: 587010
}

\begin{abstract}
The purpose of the study is to examine the process of establishing a system of comprehensive research on innovation capacity management priorities. The complexity of the concept of innovation economy potential should also be explored.

The methodological basis of the study is a systematic empirical and structural-functional approach of considering the object of research as a holistic set of interrelated elements. The scientific methods used include causal analysis.

Results of the research. The authors determined that the concept of resources as an economic category should not be equated with the concept of potential. The paper shows the structure of the concept of innovation potential, its elements and dimensions, as well as the ways of human capital formation considered as the most important component of the discussed potential. The lack of a structured theoretical discussion of the essence, meaning and ways of formation of innovative activity potential is an important problem of the entire theory of innovation.

The prospects of the research are to studying the issues of measuring innovation and human potential, taking into account information potential in order to realize the needs of innovation activity at various levels of management.

Keywords: innovation potential, human capital, organizational capabilities, management

For citation: Lavrikova N. I., Azarova N. A., Opara M. V. Formation of a system of interaction of priorities for managing the potential of innovation and human activity // Bulletin of the South Russian State Technical University. Series: Socio-economic Sciences. 2021; 14(6): 196-205. (In Russ.). http://dx.doi.org/10.17213/2075-2067-2021-6-196-205.
\end{abstract}

Введение. Формирование потенциала инновационной экономики является одной из основных целей управления инновациями, понимаемыми как создание идей, выбор, внедрение и предложение новых продуктов, услуг, систем управления и социальной организации. Это касается страны, региона и некоммерческих экономических организаций [1].

Управление инновациями означает создание таких условий, которые способствуют увеличению шансов на эффективное решение различных проблем в неопределенной среде. Оно также может применяться как в между- народном, так и в мировом масштабе, поэтому инновации связаны со способностью определять возникающие проблемы и возможности, связи между ними, выбирать их с точки зрения возможности использования и реализация. Такая реализация происходит как часть инновационного процесса, который состоит из нескольких этапов, описанных в литературе по данной теме [2-7]. Результатом инновационного процесса является конкретная инновация, которая может быть новым или модифицированным продуктом, новым технологическим процессом, новой 
системой управления или ее элементом или новым методом маркетинга.

В результате эволюции управления инновациями как приоритетной проблемы реализуется концепция инновационных систем. Выделяются Национальная инновационная система (НИС), Региональная инновационная система (РИС) и инновационные системы отдельных единиц социальной и экономической жизни. Существуют также международные инновационные системы, например, инновационная система Европейского Союза. Элементами управления национальной инновационной системой являются, например, правительство, парламент, финансовая система НИОКР или образования и т.д.

В каждой инновационной деятельности стоит выделять три блока: потенциал, процесс и эффект [8]. Цель каждого действия достичь заранее определенного эффекта, то есть до его запуска. Например, целью реализации идеи, результатом которой является новый продукт, может быть увеличение прибыли компании, увеличение клиентов, расширение рынков сбыта, экономия на расходе материалов. Инновационный процесс, состоящий из нескольких этапов, осуществляется в различных подразделениях, например в научных учреждениях, и прежде всего на предприятиях, где НИС, РИС составляют среду для их инновационной деятельности.

Очень часто как в теории, так и в экономической практике, термины «ресурс» и «потенциал» отождествляются. Однако эти понятия целесообразно различать. Ресурс может быть идентифицирован с потенциалом только тогда, когда он строго приспособлен к определенным и реализованным целям. С другой стороны, когда ресурс делает невозможным достижение целей данной деятельности, он должен быть изменен, т.е. его уровень и структура должны быть скорректированы в соответствии с поставленными целями. Неспособность согласовать ресурсы с возможностью достижения бизнес-целей приводит к неэффективности инновационной деятельности.

Методика. На этом фоне возникает вопрос, следует ли адаптировать цели к имеющимся ресурсам или необходимо ресурсы адаптировать к сформулированным целям, то есть создавать потенциал [9]?
Несомненно, в первую очередь формулируются цели, под которые адаптируются ресурсы для создания потенциала. Если бы цели были скорректированы с учетом ресурсов, то развитие оказалось бы под угрозой, или оно не состоялось бы вообще, или это было бы экстенсивное развитие. Адаптация ресурсов к сформулированным целям, т.е. создание потенциала, позволяет интенсивно развиваться. Следовательно, понятие потенциала связано с тремя элементами: ресурсом, целями и, наконец, действиями, которые позволят достичь целей. Существующие ресурсы информируют о том, что есть на самом деле, а цели сообщают о том, чего еще не существует, но через действие может возникнуть и трансформироваться в новый продукт. Таким образом, ресурс - это все, что анализируемый объект (компания, регион, страна) имеет и знает, а потенциал - это все, что компания может создать, какой новый продукт она может получить.

Результаты. Таким образом, можно заключить, что понятие потенциала следует понимать как ресурс, когда он полностью или частично трансформируется в новую желаемую ценность, указанную в цели деятельности, благодаря работе, выполняемой с использованием требуемых компетенций и навыков. Знание взаимосвязи между появлением нового товара и настоящими условиями действия позволит более эффективно согласовывать с ними потенциальные элементы в будущем. Итак, достижение целей зависит от качества имеющихся ресурсов, от способа их использования и корректировки, а также от способов достижения поставленных целей. Критериями такой корректировки могут быть:

- требования к пригодности ресурсов и навыков применительно к реализации предлагаемой инновационной деятельности, а также возможностям и угрозам, возникающим в окружающей среде [10];

- организационный потенциал, целью которого является способность использовать имеющиеся ресурсы для эффективной инновационной деятельности [11.]

Приведенные выше критерии в большей степени относятся к инновационной деятельности и показывают, что инновационная деятельность (на макро- и микроуровнях) 
требует дифференцированного потенциала. На каждом уровне цели формулируются в виде желаемых новых продуктов. На том же уровне управления, например, на уровне компаний, реализующих внедрение нового продукта в рамках отдельных этапов инновационного процесса, также требуются дифференцированные потенциалы, поскольку на каждом этапе внедрения у них разные подцели. Таким образом, потенциал - это динамическая категория.

Важнейшим элементом инновационного потенциала также является и человеческий потенциал, то есть уровень образования, знаний, навыков, опыта, инновационной культуры и прежде всего осведомленности об инновациях. На разных уровнях управления инновационной деятельностью структура вышеперечисленных потенциальных элементов различна. Несомненно, на первоначальном уровне необходим общий уровень образования с хорошим знанием принципов инновационной деятельности, закономерностей и тенденций развития цивилизации, в том числе экономического развития, и умением видеть взаимозависимость явлений. На организационном уровне важнее подготовка специалистов. Если на макроуровне определяются общие цели инновационной деятельности и направления развития, то на микроуровнях управления - методы их реализации. Это замечание касается каждой из составляющих потенциала инновационной деятельности (науки, техники, экономики). На человеческий потенциал также большое влияние оказывают здоровье, интеллектуальная и эмоциональная энергия, культура или ценности.

Информационный потенциал — это не только национальные, но и глобальные знания и их институциональные формы знания, связанные с инновационной деятельностью [12].

Технические средства как часть потенциала инновационной деятельности включают в себя всю научно-исследовательскую аппаратуру, лаборатории, машины и устройства, ИТ-оборудование, обеспечивающее быстрое межличностное общение, особенно между участниками инновационных процессов разного уровня и внутри них.

Организация и управление - важная составляющая потенциала инновационной деятельности, которая очень часто является препятствием для ее развития, а значит, требует системной модернизации. Кстати, стоит заметить, что самое главное нововведение в наше время - это внедрение новых методов организации и управления таким образом, чтобы они стали фактором, стимулирующим инновационную деятельность, а не тормозом для нее. Стоит включить вертикальные и горизонтальные связи между организациями и внутри них, международные контакты, особенно со странами ЕС в области сотрудничества в сфере науки и технологий, или тесные связи между организациями и клиентами. Важным элементом потенциала является финансово-экономическая система - методы финансирования инновационной деятельности, которая в глазах исполнителей инновационной деятельности часто является препятствием для ее развития.

Указанные компоненты потенциала инновационной деятельности и их элементы присутствуют на всех уровнях ее управления [13].

В отношении отдельных компонентов этого потенциала следует сформулировать определенные принципы управления им, независимо от уровней управления:

- принцип адаптации потенциала к сформулированным текущим и перспективным целям инновационной деятельности;

- принцип оптимальных пропорций между потенциальными компонентами;

- возможности территориального перемещения потенциала;

- принцип активизации и совершенствования стимулов для стимулирования увеличения использования потенциала инновационной деятельности.

Потенциал инновационной деятельности - это часть социально-экономического потенциала любой страны. Между этими агрегатами существует обратная связь. Социально-экономический потенциал — это среда для потенциала инновационной деятельности, которая со временем меняется согласно ускоренному развитию науки и технологий в мире.

Потенциал инновационной деятельности трансформируется в факторе ее развития, когда он будет использован в инновационном процессе, при этом потенциал по отношению 
к компаниям можно определить как совокупность активов, позволяющих генерировать инновационные идеи, их выбор и реализацию, а также коммерциализацию эффектов этих процессов. На уровне отдельных компаний наиболее важной частью инновационного потенциала, как указано выше, является человеческий капитал как часть интеллектуального капитала, который в настоящее время включает работников умственного труда. Они осуществляют три процесса [14], между которыми возникает обратная связь:

1) создание знаний;

2) распространение знаний;

3) применение знаний.

Характеристики интеллектуальных работников можно систематизировать следующим образом:

- наличие специальных знаний: академических или узкоспециализированных профессиональных;

- продвижение новых идей с точки зрения возможной их реализации;

- прямой доступ к специалистам с соответствующим опытом и обмен знаниями с теми, кто в этом нуждается;

- обеспечение работы (часто за свой счет), управление своим временем и карьерой;

- возможность работать в разных местах, в командах, на предприятиях;

- тяга к знаниям, проявляющаяся в процессе непрерывного обучения, совершенствования, повышения квалификации и переподготовки;

- новаторское отношение, видение возможности создания новых знаний;

- наличие как специальных, так и общих компетенций, способствующих сотрудничеству.

Качество человеческих ресурсов определяется на основе квалификации. Это правильный критерий решения выявленных проблем.

Высокая квалификация - весомый критерий выполнения поставленных задач, однако на будущее необходимы системные образовательные программы [15]. Отказавшись от перечисления признаков хорошей квалификации, необходимо определить условия, в которых новая команда будет управлять инновационной деятельностью. Следовательно, необходимо определить тематику курсов и тренингов, полезных для реализации прорывных инновационных проектов.

Также стоит обратить внимание на покупателей как элемент инновационного потенциала. В современных компаниях они в какой-то мере являются соменеджерами - их голос учитывается при создании новых/модифицированных продуктов или организационных систем.

Время, а точнее, умелое управление им, также может составлять элемент потенциала инновационной деятельности. Утерянное настоящее время очень часто имеет негативные последствия в будущем, поскольку время исключительный ресурс, а его запасы неэластичны. Время нельзя заменить никаким другим ресурсом. При управлении временем следует соблюдать определенные правила:

- максимально быстро реагировать на события;

- сочетать поддерживающие меры контроля, которые до сих пор были разделены и изолированы;

- поддерживать и запускать только те виды деятельности, которые создают добавленную стоимость;

- управлять производством товаров и услуг в соответствии с их конечным использованием;

- управлять социальными последствиями борьбы со временем.

Важным элементом потенциала инновационной деятельности является сотрудничество с субъектами окружающей среды, в основном субъектами сферы науки, отечественными и зарубежными предприятиями, органами местного самоуправления [16].

Потенциал инновационной деятельности также включает в себя межорганизационные проинновационные отношения, которые, как предполагается, приводят к повышению инновационности сотрудничающих предприятий, а это означает, что основная цель их создания - это создание инноваций.

Потенциалом инновационной деятельности, несомненно, является так называемый позитивный организационный потенциал, который состоит из позитивной организационной культуры и позитивного организационного климата, обуславливая поведение сотрудников в поддержку развития организации. Поскольку формирование вышепере- 
численных направлений носит стратегический характер, а инновационная деятельность также является стратегическим мероприятием, эти направления, несомненно, влияют на динамику инновационной активности.

Человеческий (кадровый) потенциал, безусловно, является важнейшей частью потенциала инновационной деятельности на уровне инновационной макросистемы, в региональных инновационных системах, на уровне микросистем на предприятиях и на уровне реализации инновационных проектов. Человеческий потенциал требует особой тщательной подготовки. Источник его приготовления находится как внутри инновационной деятельности (это эндогенные источники), так и вне ее. Экзогенными источниками формирования человеческого потенциала инновационной деятельности являются:

- система образования: высшее образование, докторантура, аспирантура; среднее образование - общее и профессиональное; зарубежные университеты;

- различные формы обучения на специализированных курсах;

- формы обмена персоналом, в том числе между странами,

- участие в реализации программ исследований и разработок, проводимых в рамках рамочных программ;

- общие и специализированные зарубежные стажировки;

- участие в различных национальных и международных конференциях;

- сотрудничество отечественных и зарубежных компаний.

Существует множество внешних источников, и формирование человеческого потенциала должно быть связано с потребностями инновационной деятельности на различных уровнях управления. Для устранения недостатков в подготовке к осуществлению инновационной деятельности на всех уровнях управления необходимо создать министерство инноваций, основной задачей которого будет координация инновационной деятельности. С другой стороны, обучение инновационной деятельности можно организовать с использованием кадров, научной литературы с результатами фундаментальных и прикладных исследований и значительного опыта на различных уровнях управления.
Обсуждение. Инновационная деятельность, как неоднократно было подчеркнуто, очень сложна, разнообразна с точки зрения уровней управления, поэтому программы обучения требуют тщательной подготовки для выполнения конкретных ролей на этих уровнях [17].

Например, в компаниях можно различать роль создателя, новатора, предпринимателя, и каждый из них в силу своих различных функций требует разного подхода к приобретению знаний и навыков и, следовательно, разного типа образования и обучение.

Кроме того, каждый этап инновационного процесса требует применения разных знаний и навыков. Однако для этого должны быть приобретены специальные и общие знания, связанные с основами инновационности и управления инновационной экономикой, чтобы затем провести специализированные теоретико-методологические исследования, относящиеся к инновационной деятельности.

Однако отправной точкой для создания кадрового потенциала для инновационной деятельности является уровень осведомленности об инновациях. Здесь стоит отметить, что модели инновационного процесса, популяризируемые в литературе, должны найти свои аналоги в выполнении ролей, которые доминируют при реализации этих моделей. Эти роли - активаторы (инициирование инновационных процессов), исследователи (информирование об исследованиях на каждой стадии инновационного процесса), создатели (создание новых инновационных и бизнес-концепций), разработчики (подготовка работ по внедрению), исполнители (занимающиеся внедрением), фасилитаторы (обеспечивающие успешную групповую коммуникацию). Правильное исполнение перечисленных ролей требует разнообразных знаний и навыков. Высшая школа (особенно школа менеджеров) должна давать базовые знания, однако специальные знания приобретаются в основном на практике.

Формирование потенциала инновационной экономики, а точнее, потенциала инновационной деятельности как комплексной проблемы, является важным элементом теории инноваций и требует более широкого теоретического осмысления. К нерешенным проблемам теории инноваций относятся и вопросы измерения этого потенциала, поскольку его 
трудно измерить количественно и не всегда измерения отражают его фактическое состояние (уровень и структуру), поэтому аналитик часто вынужден использовать описательные характеристики. Выбор индикаторов для измерения зависит, прежде всего, от правильного определения этого потенциала и целей анализа. Этими целями могут быть:

- необходимость подготовить стратегию развития компании, региона или страны;

- подача заявок в местные или национальные органы власти для поддержки инновационных процессов;

- восполнение пробелов в существующем инновационном потенциале;

- получение знаний о динамике инновационной деятельности;

- сравнение с другими отечественными или зарубежными компаниями, сравнение инновационного потенциала разных стран.

Выводы. Долгосрочное наблюдение за этими индикаторами может быть полезным для выявления определенных закономерностей в формировании потенциальных элементов или даже в установлении определенных закономерностей в этом отношении. Отправной точкой для правильного построения меры является точное определение явлений, существенных для описываемого объекта, и понимание их логики. Установленные показатели и рассчитанные на их основе показатели позволят установить (рассчитать) взаимосвязи между элементами потенциальных составляющих и эффектами инновационной деятельности и развития компании, региона и страны. Важной и пока не решенной проблемой является структура мер, позволяющих оценить способность компании к инновациям в будущем.

Предлагается классифицировать показатели инновационной активности компаний по трем критериям:

1) потенциал инновационной деятельности;

2) инновационный процесс;

3) эффекты инновационных (внедренческих) процессов.

\section{Список источников}

1. Азарова Н.А. Эффективность управления человеческим капиталом при форми- ровании регионального индекса инноваций// Регион: системы, экономика, управление. 2021. №2(53). С. 93-98.

2. Бакеев Б.В. Влияние показателей инновационного потенциала региона на результаты инновационной деятельности // Региональные проблемы преобразования экономики. 2019. №11(109). С. 147-156.

3. Дешевова Н.В. Управление инновационным развитием предприятия на основе эффективных способов мобилизации ресурсного потенциала // Инновации в образовании и науке: Межвузовский сборник научно-методических трудов / Под науч. ред. Е.Н. Атарщиковой, В.И. Бережного. М.: Русайнс, 2017. C. $98-102$.

4. Лаврикова Н.И. Влияние технологических инноваций на развитие хозяйственных систем // Международный научный журнал. 2021. №2. С. 43-51.

5. Мансурова Ю. Т. Задача минимизации инновационного риска в системе управления инновационным потенциалом предприятия // Информационные технологии интеллектуальной поддержки принятия решений (ITIDS'2019): Труды VII Всероссийской науч. конференции (Уфа, 28-30 мая 2019 г.). Уфа: Уфимский государственный авиационный технический университет, 2019. С. 83-90.

6. Омельченко И.И. Инновационный потенциал персонала и способы управления его развитием // Университетская наука региону: Материалы VI ежегодной науч.практ. конференции преподавателей, студентов и молодых ученых Северо-Кавказского федерального университета (Ставрополь, 04-22 апреля 2018 года) / Под ред. Л.И. Ушвицкого, А.В. Савцовой. Ставрополь: ООО «Издательско-информационный центр «Фабула», 2018. С. 125-127.

7. Сальников И.И. Система управления инновационной деятельностью предприятия на основе оценки его инновационного потенциала // Экономика и управление: инновационное решение проблем: Материалы международной науч.-практ. конференции (Белгород, 02-05 апреля 2019 года). Белгород: Белгородский университет кооперации, экономики и права, 2019. С. 126-135.

8. Трифонов Ю. В. Оценки инновационного потенциала и инновационной деятель- 
ности корпораций, предприятий и фирм// Управление экономическими системами: электронный научный журнал. 2019. №1(119). С. 20.

9. Шевцова Н.В. Развитие системы управления инновационной деятельностью предприятия на основе оценки его инновационного потенциала: методический аспект// Экономика и предпринимательство. 2017. №12-3(89). С. 1114-1126.

10. Bersimbayeva A.B. Marketing approach to innovation management as a tool for enhancing innovative activity of enterprises // Bulletin of Karaganda University. Economy Series. 2017. Vol. 85. №1. P. 84-90.

11. Hamdamov Sh. I. O. Methods of managing the innovation potential of the region taking Into account the key role of human capital in the transformation of resources Into innovation// Capital of Science. 2020. №10(27). P. 40-45.

12. Kuleshova Yu. V. The method of problematic presentation as a way of organizing the productive activity of students and disclosing their creativity// Наука и образование: опыт, проблемы, перспективы развития: материалы международной науч.-практ. конференции (Красноярск, 17-19 апреля 2018 года). Красноярск: Красноярский государственный аграрный университет, 2018. Р. 175-177.

13. Stroeva O.A., Lavrikova N. I., Alekhina T.A., Semenova E.E., Stepanova M.A. Development of Organizational Diagrams and Governance Mechanisms of Region's Economy// Modern Global Economic System: Evolutional Development vs. Revolutionary Leap. Lecture Notes in Networks and Systems. Springer, Cham, 2021. Vol. 198. Pp. 1807-1816.

14. Tretyakova L.A., Azarova N.A., Lavrikova N.I., Opara M.V., Tsvyrko A.A. Formation of mechanisms for creating innovative national polygons // IOP Conference Series: Earth and Environmental Science, 2021. №808(1).

15. Utegenova $\mathrm{Zh}$. S. Foreign experience of innovative capacity management // Bulletin of Karaganda University. Economy Series. 2017. Vol. 88. №4. P. 152-158.

16. Zinina O.V. Innovative education activities, features of implementation of the innovative process // Baltic Humanitarian Journal. 2020. Vol. 9. №3(32). P. 85-87.

\section{References}

1. Azarova N.A. Jeffektivnost' upravlenija chelovecheskim kapitalom pri formirovanii regional'nogo indeksa innovacij [Efficiency of human capital management in the formation of the regional innovation index]. Region: sistemy, jekonomika, upravlenie [Region: systems, economics, management]. 2021; 2(53): 93-98. (In Russ.).

2. Bakeev B.V. Vlijanie pokazatelej innovacionnogo potenciala regiona na rezul'taty innovacionnoj dejatel'nosti [Influence of indicators of innovative potential of the region on the results of innovative activity]. Regional'nye problemy preobrazovanija jekonomiki [Regional problems of economic transformation]. 2019; 11(109): 147-156. (In Russ.).

3. Deshevova N. V. Upravlenie innovacionnym razvitiem predprijatija na osnove jeffektivnyh sposobov mobilizacii resursnogo potenciala [Management of innovative development of the enterprise on the basis of effective ways of resource potential mobilization]. Innovacii v obrazovanii i nauke: Mezhvuzovskij sbornik nauchno-metodicheskih trudov [Innovations in education and science: Interuniversity collection of scientific and methodological works]. Pod nauch. red. E. N. Atarshhikovoj, V.I. Berezhnogo [In E. N. Atarshchikova, V.I. Berezhnoy (eds.)]. Moscow: Rusajns, 2017. P. 98-102. (In Russ.).

4. Lavrikova N. I. Vlijanie tehnologicheskih innovacij na razvitie hozjajstvennyh sistem [The impact of technological innovations on the development of economic systems]. Mezhdunarodnyj nauchnyj zhurnal [International Scientific Journal]. 2021; (2): 43-51. (In Russ.).

5. Mansurova Ju. T. Zadacha minimizacii innovacionnogo riska $\mathrm{v}$ sisteme upravlenija innovacionnym potencialom predprijatija [The task of minimizing innovation risk in the enterprise innovation potential management system]. Informacionnye tehnologii intellektual'noj podderzhki prinjatija reshenij (ITIDS'2019): Trudy VII Vserossijskoj nauch. konferencii (Ufa, 28-30 maja 2019 g.) [Information technologies of intellectual decision support (ITIDS'2019): Proceedings of the VII All-Russian Scientific Conference (Ufa, May 28-30, 2019)]. Ufa: Ufimskij gosudarstvennyj aviacionnyj tehnicheskij universitet, 2019. P. 83-90. (In Russ.). 
6. Omel'chenko I.I. Innovacionnyj potencial personala i sposoby upravlenija ego razvitiem [Innovative potential of personnel and ways of managing its development]. Universitetskaja nauka - regionu: Materialy VI ezhegodnoj nauch.-prakt. konferencii prepodavatelej, studentov i molodyh uchenyh Severo-Kavkazskogo federal'nogo universiteta (Stavropol', 04-22 aprelja 2018 goda) [University Science - to the region: Materials of the VI annual scientific and practical conference of teachers, students and young scientists of the North Caucasus Federal University (Stavropol, April 04-22, 2018)]. Pod red. L.I. Ushvickogo, A. V. Savcovoj [In L. I. Ushvitsky, A.V. Savtsova (eds.)]. Stavropol': OOO «Izdatel'sko-informacionnyj centr «Fabula», 2018. P. 125-127. (In Russ.).

7. Sal'nikov I.I. Sistema upravlenija innovacionnoj dejatel'nost'ju predprijatija na osnove ocenki ego innovacionnogo potenciala [The system of management of innovative activity of the enterprise on the basis of assessment of its innovative potential]. Jekonomika i upravlenie: innovacionnoe reshenie problem: Materialy mezhdunarodnoj nauch.-prakt. konferencii (Belgorod, 02-05 aprelja 2019 goda) [Economics and Management: Innovative Problem Solving: Materials of the International Scientific and Practical Conference (Belgorod, 02-05 April 2019)]. Belgorod: Belgorodskij universitet kooperacii, jekonomiki i prava, 2019. P. 126-135. (In Russ.).

8. Trifonov Ju. V. Ocenki innovacionnogo potenciala i innovacionnoj dejatel'nosti korporacij, predprijatij i firm [Evaluation of innovative potential and innovative activity of corporations, enterprises and firms]. Upravlenie jekonomicheskimi sistemami: jelektronnyj nauchnyj zhurnal [Management of economic systems: electronic scientific journal]. 2019; 1(119): 20. (In Russ.).

9. Shevcova N.V. Razvitie sistemy upravlenija innovacionnoj dejatel'nost'ju predprijatija na osnove ocenki ego innovacionnogo potenciala: metodicheskij aspekt [Development of the enterprise innovation management system based on the assessment of its innovation potential: methodological aspect]. Jekonomika i predprinimatel'stvo
[Economics and entrepreneurship]. 2017; 123(89): 1114-1126. (In Russ.).

10. Bersimbayeva A.B. Marketing approach to innovation management as a tool for enhancing innovative activity of enterprises // Bulletin of Karaganda University. Economy Series. 2017. Vol. 85. №1. P. 84-90.

11. Hamdamov Sh. I. O. Methods of managing the innovation potential of the region taking Into account the key role of human capital in the transformation of resources Into innovation// Capital of Science. 2020. №10(27). P. 40-45.

12. Kuleshova Yu. V. The method of problematic presentation as a way of organizing the productive activity of students and disclosing their creativity. Nauka i obrazovanie: opyt, problemy, perspektivy razvitija: materialy mezhdunarodnoj nauch.-prakt. konferencii (Krasnojarsk, 17-19 aprelja 2018 goda) [Science and education: experience, problems, development prospects: materials of the international scientific and practical conference (Krasnoyarsk, April 17-19, 2018)]. Krasnojarsk: Krasnojarskij gosudarstvennyj agrarnyj universitet, 2018. P. 175-177.

13. Stroeva O.A., Lavrikova N. I., Alekhina T.A., Semenova E.E., Stepanova M.A. Development of Organizational Diagrams and Governance Mechanisms of Region's Economy// Modern Global Economic System: Evolutional Development vs. Revolutionary Leap. Lecture Notes in Networks and Systems. Springer, Cham, 2021. Vol. 198. Pp. 1807-1816.

14. Tretyakova L.A., Azarova N.A., Lavrikova N. I., Opara M.V., Tsvyrko A.A. Formation of mechanisms for creating innovative national polygons // IOP Conference Series: Earth and Environmental Science, 2021. №808(1).

15. Utegenova $\mathrm{Zh}$. S. Foreign experience of innovative capacity management // Bulletin of Karaganda University. Economy Series. 2017. Vol. 88. №4. P. 152-158.

16. Zinina O.V. Innovative education activities, features of implementation of the innovative process // Baltic Humanitarian Journal. 2020. Vol. 9. №3(32). P. 85-87.

Статья поступила в редакиию 25.11.2021; одобрена после рецензирования 30.11.2021; принята к публикации 08.12.2021.

The article was submitted on 25.11.2021; approved after reviewing on 30.11.2021; accepted for publication on 08.12.2021. 


\section{ИНФОРМАЦИЯ ОБ АВТОРАХ}

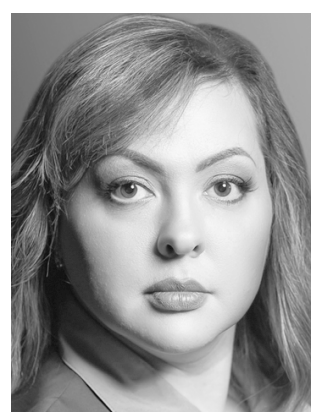

Лаврикова Наталия Игоревна - кандидат экономических наук, доцент, Академия Федеральной службы охраны Российской Федерации.

Россия, г. Орел, ул. Математика Киселёва, 2

Natalia I. Lavrikova - Candidate of Economic Sciences, Associate Professor, Academy of the Federal Guard Service of the Russian Federation.

2 Mathematika Kiseleva st., Orel, Russia

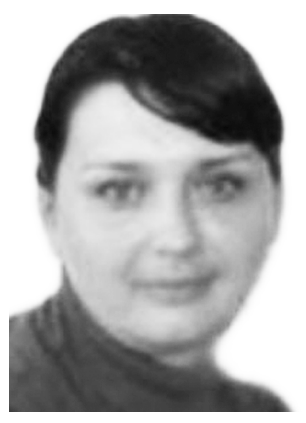

Азарова Наталья Анатольевна - кандидат экономических наук, доцент кафедры «Мировая и национальная экономика», Воронежский государственный лесотехнический университет имени Г.Ф. Морозова.

Россия, г. Воронеж, ул. Тимирязева, 8

Natalya A. Azarova - Candidate of Economic Sciences, Associate Professor, Department of World and National Economy, Voronezh State University of Forestry and Technologies named after G.F. Morozov.

8 Timiryazeva st., Voronezh, Russia

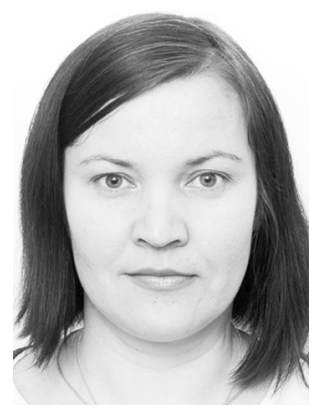

Опара Мария Васильевна - кандидат технических наук, доцент, Воронежский государственный лесотехнический университет имени Г.Ф. Морозова.

Россия, г. Воронеж, ул. Тимирязева, 8

Maria V. Opara - Candidate of Engineering Sciences, Associate Professor, Voronezh State University of Forestry and Technologies named after G. F. Morozov.

8 Timiryazeva st., Voronezh, Russia

Вклад авторов:

Лаврикова Н.И. - консультации в течение выполнения работы; итоговые выводы.

Азарова Н. А. - исследование по проблематике темы.

Опара М.В. - обзор литературных источников по исследуемой проблеме.

Contribution of the authors:

Lavrikova N. I. - consultations during the work; final conclusions.

Azarova N.A. - esearch on the problems of the topic.

Opara M. V. - review of literary sources on the problem under study. 